\title{
Exosomal miR-499a-5p Inhibits Endometrial Cancer Growth and Metastasis via Targeting VAV3
}

This article was published in the following Dove Press journal: Cancer Management and Research

\author{
Liang Jing ${ }^{1} * *$ \\ Xu Hua ${ }^{2, *}$ \\ Du Yuanna ${ }^{3, *}$ \\ Zang Rukun (DiD ${ }^{3}$ \\ Mou Junjun ${ }^{3}$ \\ 'Department of Gynecology, The \\ Affiliated Yantai Yuhuangding Hospital of \\ Qingdao University, Yantai, People's \\ Republic of China; ${ }^{2}$ Department of \\ Obstetrics, The Affiliated Yantai \\ Yuhuangding Hospital of Qingdao \\ University, Yantai, People's Republic of \\ China; ${ }^{3}$ Department of Radiotherapy, The \\ Affiliated Yantai Yuhuangding Hospital of \\ Qingdao University, Yantai, People's \\ Republic of China
}

*These authors contributed equally to this work
Correspondence: Zang Rukun;

Mou Junjun

Department of Radiotherapy, The Affiliated Yantai Yuhuangding Hospital of Qingdao University, No. 20 Yudong Road, Yantai 264000, Shandong, People's

Republic of China

Email zangrukunxiao7@I26.com;

moujunjun1986@I63.com
Background/Aim: The current therapeutic strategies for endometrial cancer are limited and unsatisfactory. Accumulating evidence suggest that microRNAs (miRNAs) participate in tumor growth and metastasis. Mesenchymal stem cells (MSCs) derived exosomes (Exos) are considered as better miRNA delivery vehicles. Here, we investigated the therapeutic effect of exosomal miR-499a-5p (miR-499) in human endometrial cancer metastasis.

Methods: Microarray analysis and RT-PCR were performed to detect the relative expression of miR-499 in endometrial cancer tissues and cell lines. MSC-derived Exos were characterized by transmission electron microscope (TEM), Western blot (WB), and nanoparticle tracking analysis (NTA). miR-499 was loading into Exos using electroporation. Cell proliferation and angiogenesis capacity were tested by 5-ethynyl-29-deoxyuridine (EdU) assay and tube formation assay, respectively. Dual-luciferase reporter assay (DLR) was used to confirm the connection of miR-499 and VAV3.

Results: We found that the expression of miR-499 was significantly downregulated in cancer tissues compared with adjacent tissues in endometrial cancer patients. Moreover, exosomal miR-499 not only dramatically suppressed endometrial cancer cells proliferation, endothelial cells tube formation in vitro, but also inhibited tumor growth and angiogenesis in vivo. In addition, we confirmed that miR-499 directly targets the 3'UTR sequence of VAV3.

Conclusion: The novel identified exosomal miR-499 functions as a tumor suppressor in endometrial cancer though regulating VAV3, and these findings could be a valid molecular target for endometrial cancer therapy.

Keywords: endometrial cancer, exosome, miR-499a-5p, VAV3, angiogenesis

\section{Introduction}

Endometrial cancer is one of the most common malignancies in women and the morbidity of endometrial carcinoma exhibits rapid growth around the world in recent years. ${ }^{1}$ Based on clinicopathologic feature and pathogenesis, endometrial cancer can be broadly classified into estrogen dependent (type I) and non-estrogen dependent (type II). ${ }^{2}$ In China, 80 90\% of newly diagnosed endometrial cancer cases are classified as type $\mathrm{I}^{3}$ In the majority of type I cases, patients are often diagnosed at an early stage. With standard surgical treatment and following chemotherapy, the 5-year relative survival rate of these patients is approximately $82 \%{ }^{4,5}$ However, some patients with recurrent endometrial carcinoma could not get a satisfactory therapeutic effect from current therapies. The poor prognosis of these patients is mainly due to malignant proliferation and distant metastasis. ${ }^{6,7}$ To date, the underlying mechanism of endometrial cancer proliferation and distant 
metastasis remains uncertain. It is worth noting that growing findings have suggested that miRNA aberrant may play a crucial role in this pathophysiological process. Therefore, miRNA targeted therapy may be a great potential practical approach in endometrial cancer therapy.

miRNAs are highly conserved non-coding RNAs. The mature form of miRNAs have become considered as crucial regulators in gene expressions. Accumulating evidence suggests that miRNAs are involved in multiple biological processes, including proliferation, apoptosis, and metabolism. ${ }^{8}$ Furthermore, aberrant expression of miRNAs has been reported in many cancers, and these miRNAs participate in tumor growth, tumor angiogenesis, distance metastasis, and immune evasion by regulating the expression of the target genes. ${ }^{9,10}$ Several miRNAs, including miR-134, miR-148b, miR-183, and miR-625 have been studied for their functional role during the endometrial cancer pathophysiological process. ${ }^{11-14}$ Among these miRNAs, miR-499 is particularly noteworthy, because some reports indicate that miR-499 involved in the angiogenic activity of vascular endothelial cells. ${ }^{15}$ However, the exactly biological function of miR499 in malignant proliferation and distant metastasis of endometrial cancer is still unclear.

MSCs have been applied to treat various diseases. The wide use of MSCs was due to their properties, such as immune privilege and capacity to proliferation at the lesion sites. ${ }^{16}$ Recent studies reveal that MSCs mediate the therapeutic benefits through paracrine effect rather than cellular manner. ${ }^{16}$ MSCs secreted Exos play a major role in cell-to-cell communication by transfer cargo including lipids, proteins, mRNA, and miRNA molecules. ${ }^{17}$ Accumulating evidence suggests that MSCs have antitumor effects, and MSC-derived Exos may have similar therapeutic functions as MSC. ${ }^{17}$ Compared to MSCs, MSC-derived Exos have numerous benefits, including exceptional stability features, easy to storage, delivery, and management. ${ }^{18}$ Thus, Exos therapy would be a promising therapeutic strategy for endometrial cancer patients, which could be further optimized for its clinical application.

In the current study, we investigated the function of exosomal miR-499 on endometrial cancer. Our findings showed that miR-499 was downregulated in endometrial cancer tissues by microarray analysis and RT-PCR. We also found that exosomal miR-499 suppressed tumor growth and angiogenesis in endometrial cancer. In addition, our results indicated that the antitumor effect of miR-499 is achieved by directly targeting VAV3.

\section{Materials and Methods Ethical Statement}

This study was approved by the Ethics Committee of the Affiliated Yantai Yuhuangding Hospital of Qingdao University. All participants were informed of the details of the study design and signed the informed consent form. All animal experiments performed in the current study were in accordance with the Guide for the Care and Use of Laboratory Animal by International Committees.

\section{Human Endometrial Cancer Specimen Collection}

Tumor tissues and the adjacent noncancerous tissues from five endometrial cancer patients were collected from Yantai Yuhuangding Hospital between January 1, 2018 and December 31, 2019. The inclusion criteria were as follows: primary diagnosed with endometrial cancer, underwent surgery without prior radiotherapy, chemotherapy, or molecular targeted therapy. The tumor type and tumor node, metastasis (TNM) stage of each specimen were histologically determined according to the classification system established by the World Health Organization (WHO). All samples were stored in liquid nitrogen for further detection and analysis.

\section{MicroRNA Microarray}

Total RNA of the tumor samples were obtained using TRIzol reagent (Invitrogen, Carlsbad, CA, USA) according to the manufacturer. The quantity of RNA samples were examined by the Agilent 2100 Bioanalyser (Agilent, Chengdu, China), and RNA integrity was verified by gel electrophoresis. miRNA expression analysis was performed using the RiboArray platform as described previously. This microarray platform is designed based on a locked nucleic acid (LNA) technology. Differentially expressed miRNAs were identified through fold change filtering. Statistical analysis using $T$ test, and $P$-value $<0.05$ and fold change $>2$ were considered significantly differentially expressed. Microarray images were generated by $\mathrm{R}$ software.

\section{Cell Culture}

MSCs were isolates from C57/BL6 mice (male, 6 weeks old, supplied by Nanjing University Medical Animal 
Laboratory Center, China) as previously published. ${ }^{19}$ MSCs identification was performed by flow cytometry. Monoclonal antibody CD29, CD90, CD34, and CD45 (Invitrogen) were used for verification of cell surface antigens. Human normal endometrial stromal cells (SHT290) and human endometrial cancer cell lines, including Ishikawa, HEC1A, and AN3CA, were purchased from American Type Culture Collection (ATCC, VA, USA). Human umbilical vein endothelial cells (HUVEC) were purchased from Cell \& Biological Engineering Co., Ltd. (CELLBIO, Shanghai, China). As per the instructions, MSCs, SHT290, Ishikawa, HEC1A, and AN3CA were cultured in Dulbecco's modified Eagle's medium (DMEM, HyClone, UT, USA) containing 10\% fetal bovine serum (FBS, Gibco, MD, USA) in a humid atmosphere containing $5 \% \mathrm{CO}_{2}$ at a temperature of $37^{\circ} \mathrm{C}$. HUVECs were cultured in EGM-2 (Lonza, MD, USA) supplemented with 5\% FBS in a humid atmosphere containing $5 \% \mathrm{CO}_{2}$ at a temperature of $37^{\circ} \mathrm{C}$.

\section{MSC-Derived Exos Isolation and Identification}

MSCs in logarithmic growth phase were cultivated in DMEM supplemented with 10\% Exos-free FBS for 48 hours before culture medium collection. The supernatant was then under through an ultracentrifugation (100,000 $\mathrm{g}$ for 120 minutes at $4^{\circ} \mathrm{C}$ ) for Exos extraction. Purified Exos were diluted in phosphate buffer saline (PBS) and stored at $-80^{\circ} \mathrm{C}$ for the following experiments. The morphological features, specific markers, and particle diameter of extracted Exos were detected by transmission electron microscope (TEM, Thermo Fisher Scientific, CA, USA), Western blot and Nanoparticle tracking analysis (NTA, Thermo Fisher Scientific) according to the manufacturer's instructions.

\section{Exos Loading and Cell Grouping}

The oligonucleotides were synthesis by RiboBio Co., Ltd. (RiboBio, Guangzhou, China). Exos were loaded with miR-499 using electroporation performed on a Gene Pulser II system (Bio-Rad, CA, USA) according to the manufacturer's instructions. In brief, Exos $(40 \mu \mathrm{g})$ and miR-499 (1000 nM) were mixed in $1 \mathrm{~mL}$ transfection buffer, and the mixture was electroporated using a pulse $20 \mathrm{~ms}$ and 500 voltage. Then the transfection tube was incubated at $4^{\circ} \mathrm{C}$ overnight. After washed with PBS, the Exos were diluted in phosphate buffer saline (PBS) and stored in $-80^{\circ} \mathrm{C}$ for the following experiments. Before cell transfection, Exos were labeled with DiI lipophilic dye (Invitrogen) as described previously. Exos internalized by cell were observed under an inverted fluorescence microscope (Leica DMI6000B, Germany). To investigate the function of exosomal miR-499 on endometrial cancer metastasis, cell were assigned into the Scramble group, miR-499 mimics group, and miR-499 inhibitor group. Cells $\left(4 \times 10^{4}\right.$ cells/well $)$ in logarithmic growth phase were co-cultured with $5 \mu \mathrm{g}$ Exos in a 6-well-plate for 6 hours. Then these cells were collected for follow-up experiments.

\section{EdU Cell Proliferation Assay}

EdU assay was performed using a Click-iT EdU assay kit (Invitrogen), as described previously. ${ }^{20}$ Briefly, Ishikawa cells $\left(2 \times 10^{3}\right.$ cells/well) or HUVECs $\left(1 \times 10^{4}\right.$ cells/well $)$ were seeded and cultured in 12 -well plates at $37^{\circ} \mathrm{C}$. After 48 hours incubation, EdU solution $(20 \mathrm{mM})$ was added to the cell culture media. For another 2 hour incubation, cells were fixed with 4\% paraformaldehyde (PFA, Invitrogen). DAPI (1:1,000, Invitrogen) were used for nuclear stain. EdU positive cells were detected and calculated under an inverted fluorescence microscope (Leica DMI6000B, Wetzlar, Germany). All experiments were performed three times in triplicate.

\section{Tube Formation Assay}

Tube formation assay was carried out using Matrigel matrix (Corning, NY, USA) as described previously. ${ }^{19}$ Briefly, 96-well plates were coated with Matrigel matrix (100 $\mu \mathrm{L} /$ well) and incubated at $37^{\circ} \mathrm{C}$ for 30 minutes to make the Matrigel matrix coagulation. HUVECs $\left(2 \times 10^{4}\right.$ cells/well) were then seeded on the 96-well plates. After 8 12 hours incubation, the tube length and tube mashes were observed and calculated under an inverted microscope (Leica DMI6000B). All experiments were performed three times in triplicate.

\section{Xenograft Experiments}

Animal experiments in the current study were approved by our Institutional Laboratory Animals Committee, and conducted following The Guidelines for Laboratory Animal Care and Use by the Affiliated Yantai Yuhuangding Hospital of Qingdao University. A total 16 BALB/c nude mice (male, weight 20-25 g) were supplied by the Nanjing University Medical Animal Laboratory Center, China. Xenograft experiments were performed as 
described previously. ${ }^{21}$ In brief, mice were anesthetized using sodium pentobarbital $(20 \mathrm{mg} / \mathrm{kg})$. Ishikawa cells $\left(5 \times 10^{6}\right)$ were suspended with $0.2 \mathrm{~mL}$ serum-free EGM-2 media and Matrigel matrix (v/v 1:1) and then injected onto the back of nude mice under sterile conditions. Blank Exos or exosomal miR-499 was intratumorally injected once every 3 days. Tumor volume was calculated according to the formula: $\mathrm{V}=\mathrm{width}^{2} \times$ length $\times 0.5$. Mice were sacrificed on the 28th day. Xenograft tumor samples were sliced into $4 \mu \mathrm{m}$ sections for immunofluorescence staining.

\section{Immunofluorescence Staining}

To investigate the capillary density, xenograft tumor sections were immune-stained with mouse specific antibody CD31 (1:500, Abcam, MA, USA). Goat anti-mouse Alexa fluor 555 $(1: 2,000$, Abcam) was used as a second antibody. The number of microvessels was observed and calculated under an inverted fluorescence microscope (Leica DMI6000B).

\section{DLR Assay}

VAV3 wild type or mutated 3'-UTR sequence containing the putative binding sites of miR-499 was cloned into the pmirGLO vector (Ruibo, Guangzhou, China). Cells (Ishikawa or HUVECs) were plated in 24-well plates and co-transfected with the recombinant plasmids together with miR-499 mimics or negative control (NC) using Lipofectamine 3000 (Invitrogen). The luciferase activity was measured by Dual-Luciferase Assay Kit (Promega, WI, USA) after 48 hours transfection according to the instructions.

\section{RT-PCR Analysis}

RT-PCR analysis was performed as previous published. ${ }^{18}$ Total RNA was obtained using TRIzol reagent (Invitrogen) according to the manufacturer. Reverse transcription was performed using the PrimeScript ${ }^{\circledR}$ miRNA cDNA Synthesis kit (TaKaRa, Dalian, China) for miRNAs or the PrimeScript ${ }^{\circledR}$ RT reagent kit (TaKaRa) for general genes. RT-PCR was conducted using SYBR Green qPCR Master Mix kit (Thermo Scientific, Shanghai, China) and performed on the Applied Biosystems 7500 Real-Time PCR System (Applied Biosystems, CA, USA). U6 and GAPDH were served as internal controls. The relative expression levels of target genes was calculated using $2^{-\Delta \Delta} \mathrm{Ct}$ method. The primers used are listed in Table 1. All experiments were performed three times in triplicate.
Table I The Sequences of the Primers

\begin{tabular}{|l|l|}
\hline \multirow{2}{*}{ Gene Name } & Sequence $\left(\mathbf{5}^{\prime} \mathbf{-}-\mathbf{>} \mathbf{3}^{\prime} \mathbf{)}\right.$ \\
\hline \multirow{2}{*}{ VAV3 } & GGTCCAGACTGGGGTCCCAGC \\
\cline { 2 - 2 } & GCATGCCGCAGTGGTTAGGGA \\
\hline \multirow{2}{*}{ U6 } & TGTGTGCCCAAACCAGTAG \\
\cline { 2 - 2 } & AAGTTCGGTCTCTGCTTGC \\
\hline \multirow{2}{*}{ GAPDH } & CTCGCTTCGGCAGCACA \\
\cline { 2 - 2 } & AACGCTTCACGAATTTGCGT \\
\hline & GCACCGTCAAGGCTGAGAAC \\
\cline { 2 - 2 } & TGGTGAAGACGCCAGTGGA \\
\hline
\end{tabular}

\section{Western Blot Analysis}

Western blot analysis was performed as previous published. ${ }^{18}$ Then $30 \mu \mathrm{g}$ Exos protein were separated with sodium dodecyl sulfate-polyacrylamide gel electrophoresis (SDS-PAGE) and transferred onto polyvinylidene difluoride PVDF membranes (Millipore, MA, USA). Primary antibodies, including CD9 $(1: 1,000$, BD Biosciences, NJ, USA) and TSG101 (1:1,000, BD Biosciences) were used for incubation with the PVDF membranes at $4^{\circ} \mathrm{C}$ overnight. The PVDF membranes were then incubated in the secondary antibody for 1 hour at RT. The membrane was developed by ECL Plus reagents (Thermo Scientific) and exposed under the ChemiDoc XRS system (Bio-Rad). All experiments were performed three times in triplicate.

\section{Statistical Analysis}

All parameters were represented as the mean \pm SD from at least three independent experiments. One way ANOVA and two way ANOVA were performed to compare the differences of two groups and multiple groups, respectively. Multiple comparisons among the groups were performed using Student-Newman-Keuls (S-N-K) method. The difference was considered significant as a $P$-value is less than $0.05(P<0.05)$. GraphPad Prism 6.0 software (GraphPad Software, CA, USA) and R language was used for statistical analysis and images generation.

\section{Results}

\section{miR-499 Was Downregulated in}

\section{Endometrial Cancer}

To identify the differentially expressed miRNAs in endometrial cancer, we collected tumor tissues and adjacent 
noncancerous tissues from five patients who had been diagnosed with endometrial cancer and performed microarray analysis on these specimens. Pathological examination showed that all the five cases were endometrioid adenocarcinoma. With the inclusion criterion $|\log \mathrm{FC}|>2$, $P$-value $<0.05,68$ differentially expressed miRNAs were found. The heatmap exhibited the top 20 differentially expressed miRNA between normal tissues and endometrial cancer tissues (Figure 1A). Among these miRNAs, miR-499 was one of the most prominently downregulated miRNAs in endometrial cancer tissues. Furthermore, we employed three miRNA prediction databases (TargetScan, miRDB, and miRWalk) to investigate the target gene of miR-499. As shown in the Venn diagrams (Figure 1B), 105 genes were found in the intersection. Among these mRNAs, VAV3 has been reported to be involved in endometrial cancer metastasis. Therefore, we selected VAV3 for further study. To verify the result of microarray, we examined the expression level of miR-499 and VAV3 in endometrial cancer cell lines (Ishikawa, HEC1A, and AN3CA) by RT-PCR. We found that miR-499 was dramatically downregulated while VAV3 was up-regulated in endometrial cancer cells (Figure 1C). These results manifest that miR-499 may play a crucial role in endometrial cancer development and metastasis.

\section{Characterization of MSC and MSC-Derived Exos}

We isolated MSCs from young C57/BL6 mice as described previously. ${ }^{19}$ As shown in Figure 2A, MSCs exhibited a typical spindle-shaped morphology. Flow cytometry was used to further confirm the expressed phenotypic markers of MSCs. The result showed that the isolated cells were highly expressed CD29 and CD90, but were negative for CD34 and CD45 (Figure 2B). This result is consistent with the criteria of International Cell Therapy Association (ISCT) for MSC identifying. Next, we collected Exos from MSC culture medium by ultracentrifugation. TEM analysis exhibited the morphology of Exos to be a classical "cup-shaped" (Figure 2C). We analyzed the expression of exosomal markers of these isolated particles with Western blotting. As shown in Figure 2D, CD9 and TSG101 were present in Exos but not in cells. Furthermore, NTA analysis demonstrated that the diameters of these particles mainly ranged from 40$110 \mathrm{~nm}$ and peaked at $100 \mathrm{~nm}$ (Figure 2E). The results demonstrate that the characteristics of isolated Exos in our study met the classical criteria for Exos.

\section{Electroporation Efficiency Loaded miR-499 in Exos}

MSC-derived Exos were loaded with miR-499 with Gene Pulser II system as described previously. ${ }^{19}$ As exhibited in Figure 3A, the expression level of miR-499 significantly increased in Exos after electroporated with miR-499 mimics. On the contrary, miR-499 inhibitors electroporation decreased the expression level of miR-499 in Exos. To verify the uptake of Exos by endometrial cancer cells, Exos were labeled with fluorescent carbocyanine dyes CM-Dil (red) and incubated with cells in vitro. DAPI (blue) were used for nuclear stain post- 6 hours incubation. Using
A

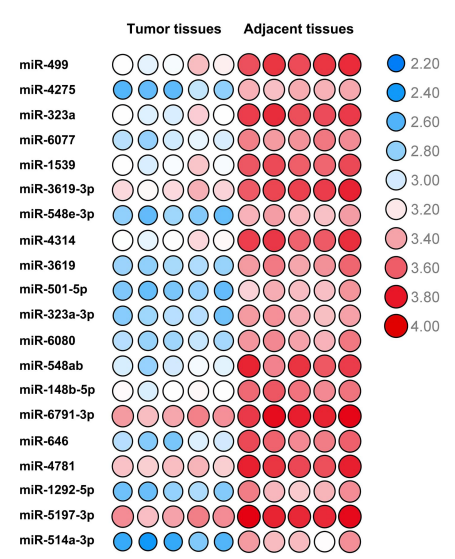

B

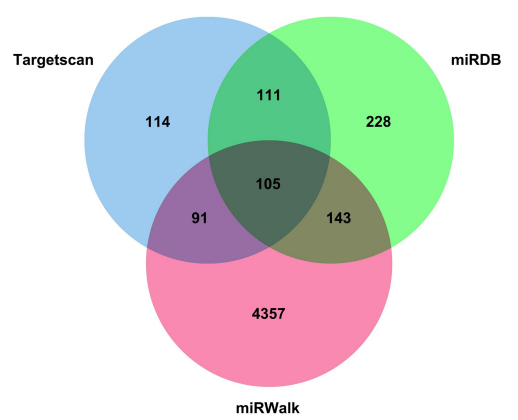

C

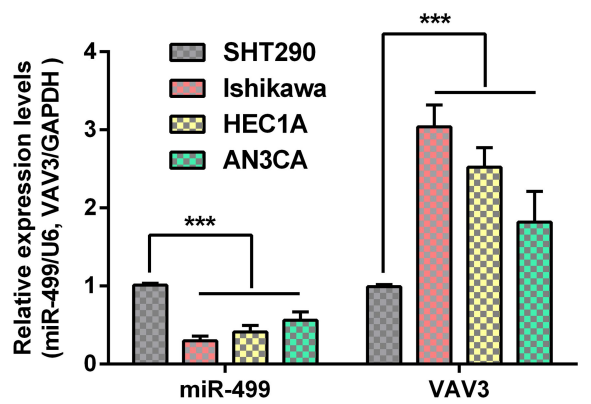

Figure I Gene expression profile in endometrial cancer. (A) Hierarchical clustering image of different expressed miRNAs between endometrial cancer tissues and adjacent normal tissues from patients. (B) Venn diagram was generated to exhibit putative target gene of miR-499 from prediction database, including TargetScan, miRDB, and miRWalk. (C) miR-499 and VAV3 expression in endometrial cancer cells and normal endometrium cells ( $n=3$ each group). Data are presented as the means \pm SD. $* * * P<0.0001$ 
A

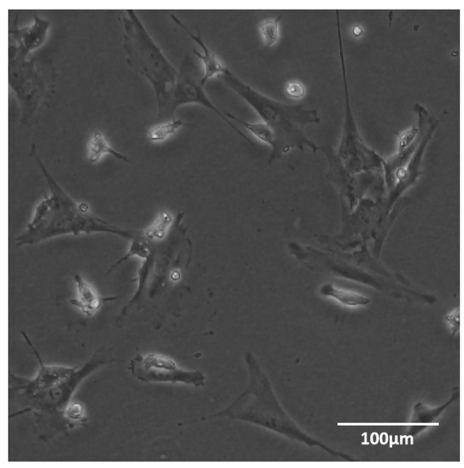

C

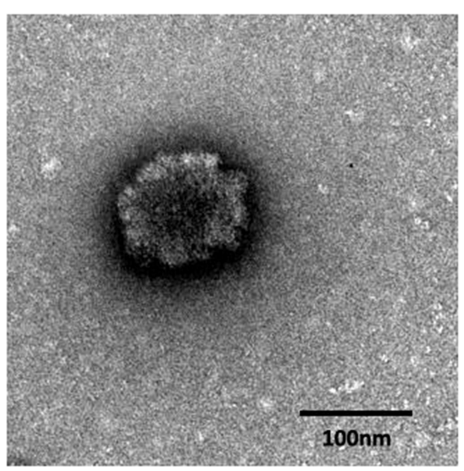

B
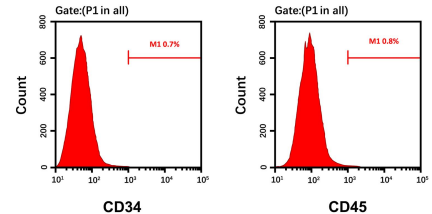

CD45
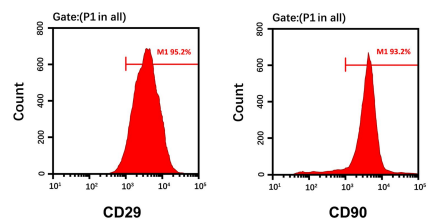

D

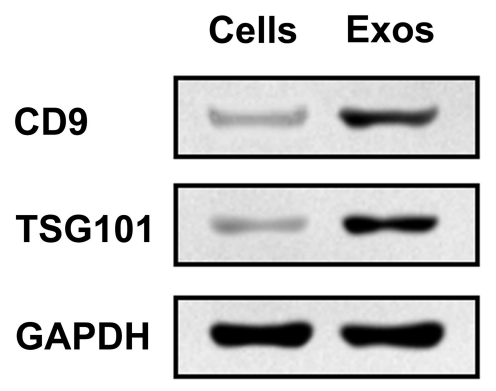

E

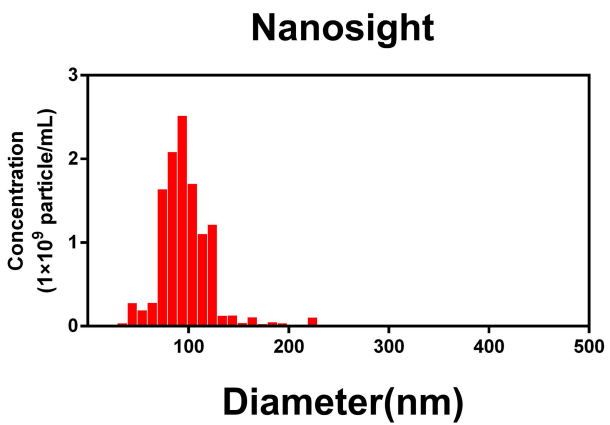

Figure 2 Identification of MSCs and MSC-derived Exos. (A) Morphological analysis of MSCs. Scale bar=100 $\mu \mathrm{m}$. (B) Flow cytometry results showed that MSCs positive expressed CD29, CD90 but negative expressed CD34, CD45. (C) Morphological analysis of MSC-derived Exos. Scale bar=100 nm. (D) MSC-derived Exos positive expressed CD9 and TSGI0I. (E) NTA analysis showed that the diameter of MSC-derived Exos mainly ranged from 80-I20 nm.

a fluorescence microscope, we provided qualitative evidence that the Exos were successfully absorbed by endometrial cancer cells, as displayed in Figure 3B. Further RT-PCR analysis result exhibited that a significant increase of miR-499 expression in endometrial cancer cells and HUVECs taking up Exos loaded with miR-miR-499 mimics when compared with negative controls and cells taking up Exos loaded with miR-499 inhibitor (Figure 3C and D). These results reveal that electroporation is an efficiency method for miRNA transfer.

\section{Exosomal miR-499 Suppresses Endometrial Cancer and HUVECs Proliferation}

In this study, we investigate the function of exosomal miR499 on proliferation in endometrial cancer cells (Ishikawa) and endothelial cells (HUVECs) using EdU cell proliferation assay. We first performed the EdU cell proliferation assay in Ishikawa cells. Our results showed that the EdU-positive expression cell rate was significantly decreased in the exosomal miR-499 mimics groups when compared with the blank Exos groups and exosomal miR-499 inhibitors groups (Figure 4A and B). Next, we performed the EdU cell proliferation assay in HUVECs and similar results were observed (Figure 4A and C). These findings manifest that exosomal miR-499 suppresses cell proliferation both in endometrial cancer cells (Ishikawa) and endothelial cells (HUVECs).

\section{Exosomal miR-499 Suppresses Angiogenesis in vitro}

The effects of exosomal miR-499 on angiogenic behavior of HUVECs in vitro were detected via Tube formation assay. HUVECs were incubated with blank Exos, miR499 mimics electroporated Exos, and miR-499 inhibitors electroporated Exos. The tube length and the number of tube meshes were calculated under an optical microscope. Our results showed that the tube length and number of tube meshes were significantly reduced in miR-499 mimics electroporated Exos groups. On the contrary, the tube length and number of tube meshes presented a significant increase in miR-499 inhibitors electroporated 
A

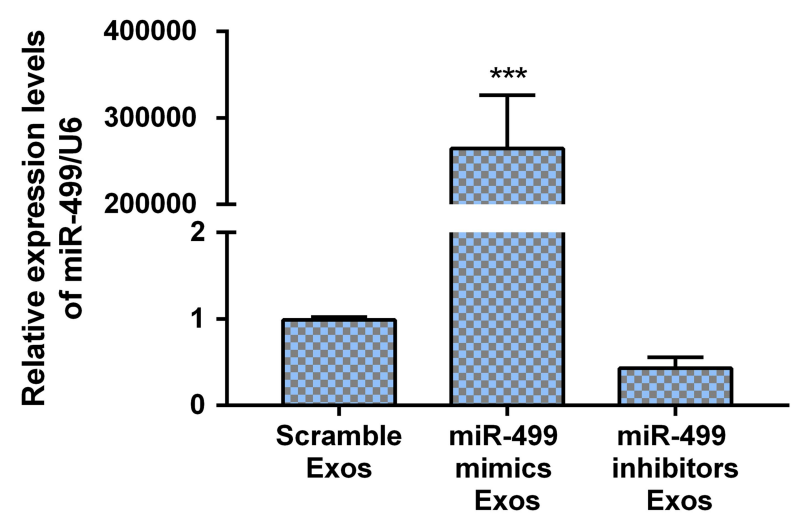

C

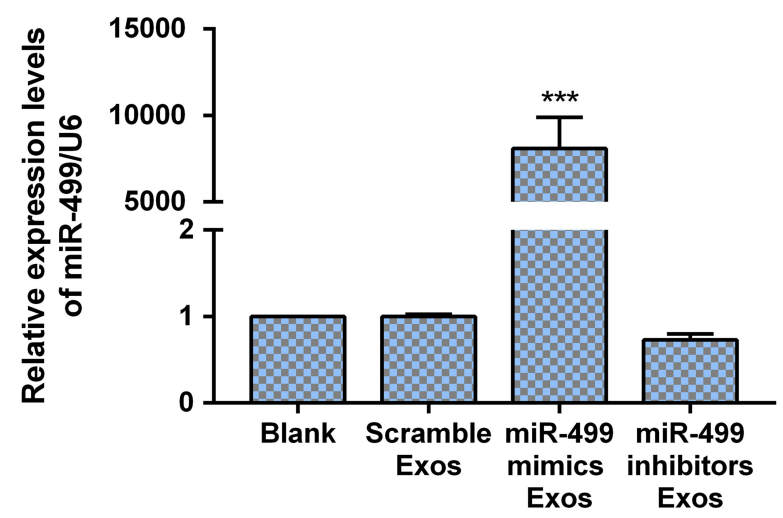

Ishikawa
B

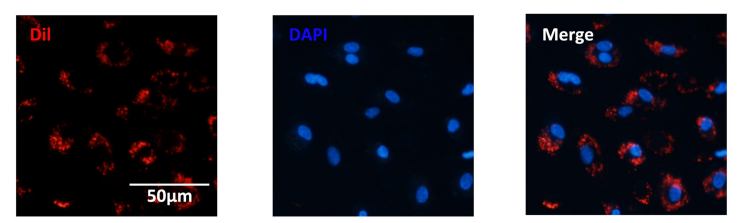

D

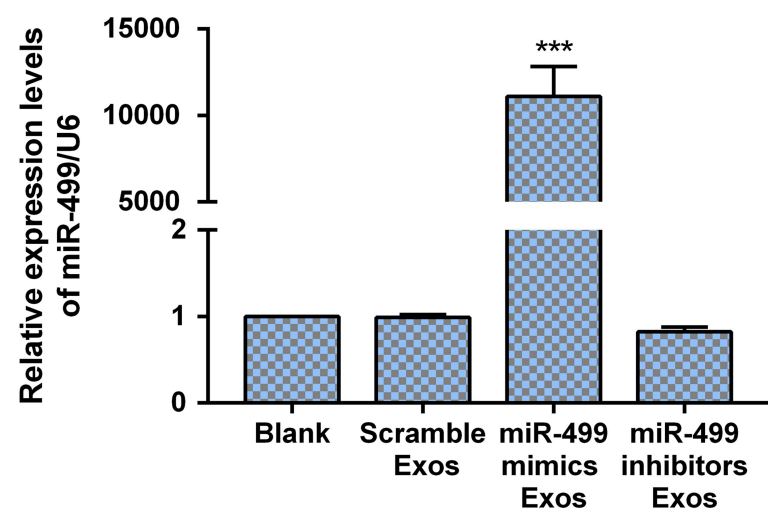

HUVECs

Figure 3 Electroporation efficiency loaded miR-499 in Exos. (A) miR-499 expression was dramatically increased in Exo after miR-499 mimics electroporation ( $\mathrm{n}=3$ each group). (B) Dil-labeled Exos were internalized by HUVECs. Scale bar=50 $\mu \mathrm{m}$. (C and D) miR-499 expression was dramatically increased in Ishikawa and HUVECs after cocultured with exosomal miR-499 $\left(\mathrm{n}=3\right.$ each group). ${ }^{* * *} p<0.0001$.

Exos groups (Figure 5). As evidenced by tube formation assay, exosomal miR-499 suppressed the proangiogenic ability of HUVECs and this effect may be involved in the pathophysiology of endometrial cancer metastasis.

\section{Exosomal miR-499 Inhibits Tumor Growth and Angiogenesis in vivo}

Previous sections showed that exosomal miR-499 suppressed cells proliferation and angiogenesis in vitro. To further verify the therapeutic effect of exosomal miR-499 on endometrial cancer, we examined the weight, volume, and vessel density of xenograft tumor. RT-PCR were performed to test the expression of miR-499 in xenograft tissues on day 28 after the model was established. Our result showed that the expression of miR-499 obviously increased in the exosomal miR-499 group when compared with the blank Exos group (Figure 6A). The xenograft tissues weight and volume were measured to evaluate the growth of tumor. Compared with the blank Exos group, the tumor weight and volume were dramatically decreased in the exosomal miR-499 group (Figure 6B-D). Furthermore, capillary density of xenograft tissues was also examined. As presented in Figure 6E and F, compared with the blank Exos group, the tumor tissues in the exosomal miR-499 group had a lower capillary density. These findings manifest that exosomal miR-499 effectively suppresses tumor growth and angiogenesis in vivo.

\section{miR-499 Directly Targets on VAV3}

To further understand the underlying mechanism of miR499 in endometrial cancer development and metastasis, we performed DLR assays both in Ishikawa cell line and HUVECs. Presumptive binding sites of miR-499 on the pmirGLO-VAV3-WT and pmirGLO-VAV3-Mut are shown (Figure 7A). As presented in Figure $7 \mathrm{~B}$ and $\mathrm{C}$, the 


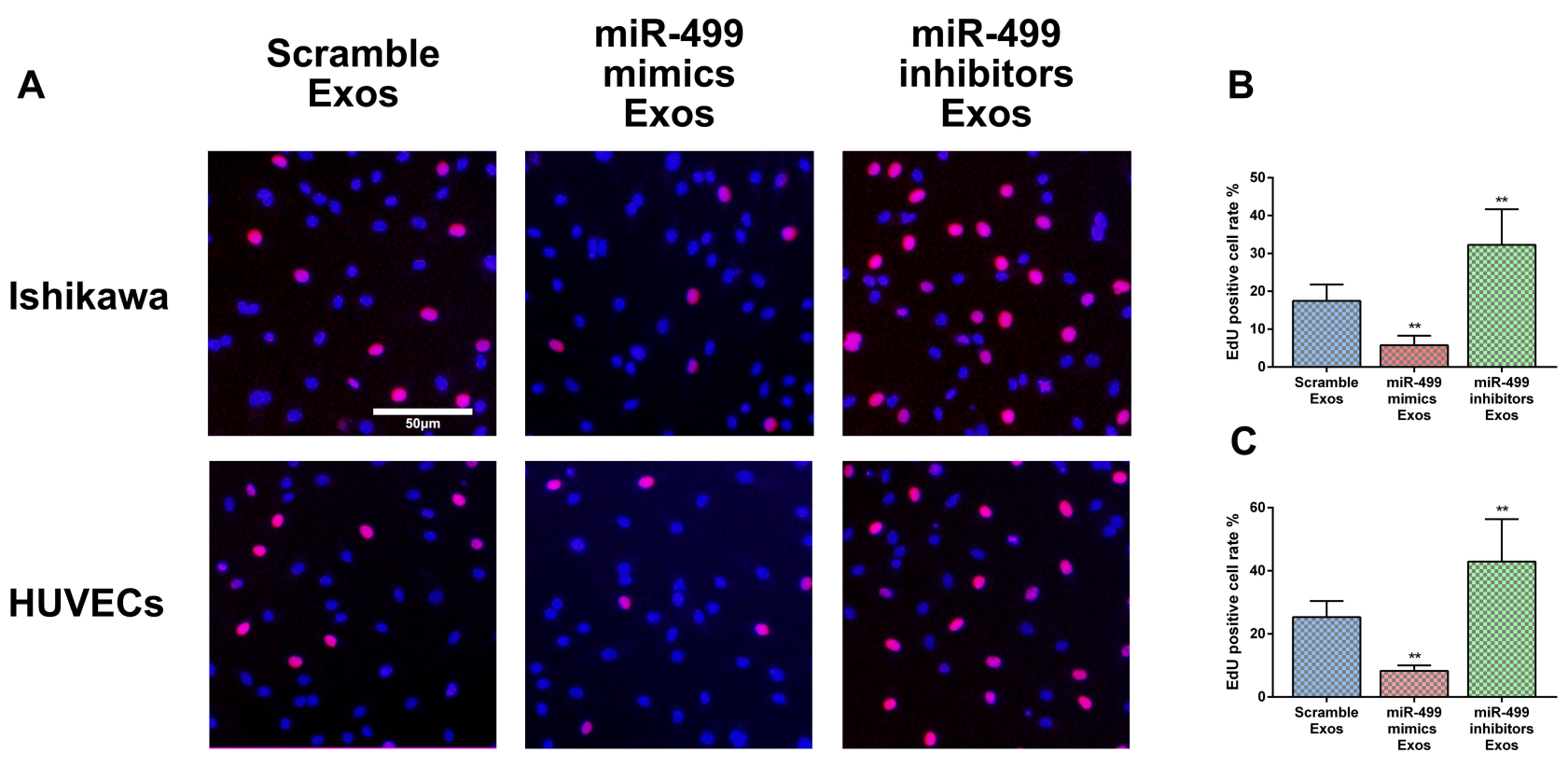

Figure 4 miR-499 inhibited cell proliferation. (A) EdU positive cell were observed under an inverted fluorescence microscope. Scale bar=50 $\mu$ m. (B and C) Statistics of EdU positive cells in Ishikawa and HUVECs ( $\mathrm{n}=3$ each group). $* * p<0.001$.

luciferase activity in the pmirGLO-VAV3-WT group was dramatically decreased when transfected with miR-499 mimics, while the luciferase activity in the pmirGLOVAV3-Mut group had no changes in cells transfected with miR-499 mimics. These results confirm that VAV3 has a directly binding site of miR-499.

\section{Discussion}

Gene and molecular therapy has made great strides during the last decade. miRNAs are expected to be novel therapeutic agents, especially for the treatment of cancer. miRNAs induce endogenous gene-silencing, resulting in the knockdown of the targeted series of proteins regulating specific signaling pathways directionally. Therefore, miRNAs have attracted much attention as therapeutic molecules to treat certain cancers with genetic mutations. In the current study, we investigate the potential therapeutic effect of exosomal miR-499 on endometrial cancer. We firstly observed that the expression level of miR-499 was notably downregulated in both endometrial cancer tissues and cell lines when compared with normal non-cancer tissues and cells. Moreover, we revealed that exosomal miR-499 significantly inhibited endometrial cancer cells proliferation and endothelial cells tube formation in vitro. On the other hand, we also demonstrated that exosomal miR-499 inhibited tumor growth and angiogenesis in vivo. Finally, DLR assay confirms that VAV3 contains a direct binding site for miR-499. These findings indicate that
miR-499 gives an inspiring hope of therapeutic molecules for the clinical treatment of this dangerous malignancy.

Endometrial cancer is one of the most common types of gynecological malignancy among women, and its morbidity has been increasing in the past decades around the world. ${ }^{1}$ A large proportion of endometrial cancer patients can be diagnosed at an early stage and receive a timely surgical treatment, the 5 -year survival rate of these patients estimated higher than $90 \%{ }^{1}$ However, the patients diagnosed with stage III and IV endometrial cancer still suffer from poor prognosis, which is associated with the frequent recurrence and metastasis. Despite various pharmacological agents having been designed to treat this disease, the overall 5-year survival rate of these late stage patients with metastatic tumor was estimated to be lower than $20 \%{ }^{7}$ Therefore, it is necessary and urgent to explore the pathological mechanisms, and develop a new effective strategy for metastatic endometrial cancer treatment.

It is generally accepted that cancer cell proliferation and tumor angiogenesis is the critical event in tumor recurrence and metastasis. Thus, it is of great significance to figure out the specific molecular mechanism involved in the proliferation and tumor angiogenesis of endometrial cancer. Accumulating evidences suggest that miRNAs are involved in multiple physiological processes, including cell differentiation, apoptosis, proliferation, embryonic development, and stem cell renewal. ${ }^{9}$ Moreover, aberrant miRNA 


\section{A Scramble Exos}

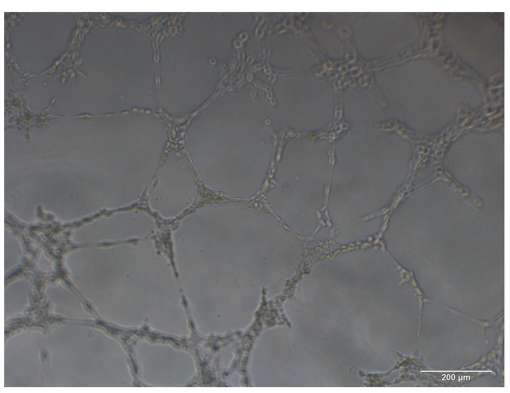

miR-499

mimics Exos

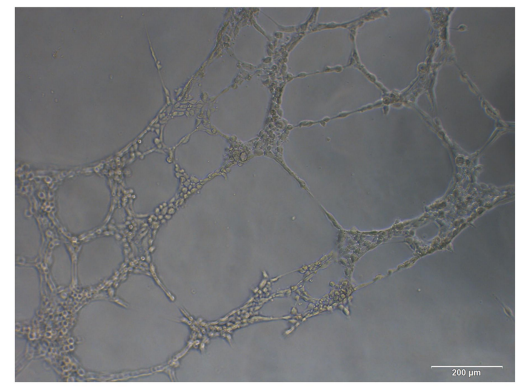

\section{miR-499 \\ inhibitors \\ Exos}

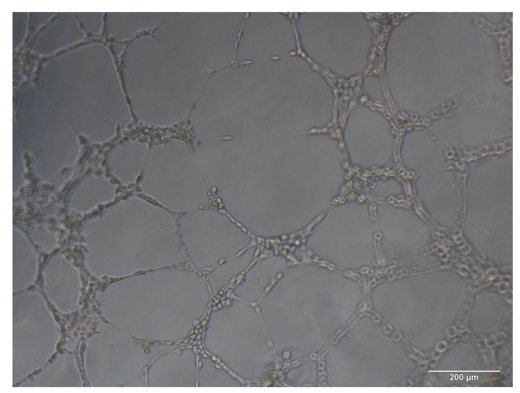

B

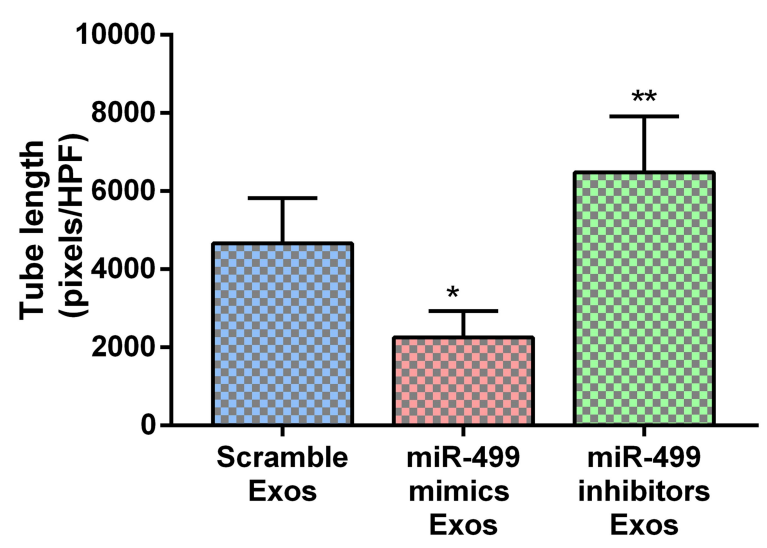

Tube Length
C

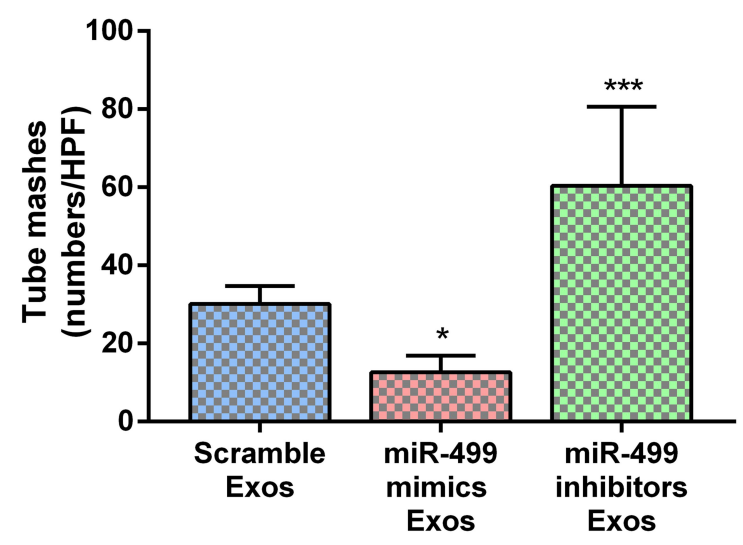

Tube Mashes

Figure 5 miR-499 inhibited tube formation in vitro. (A) Tube composed of HUVECs were observed under an optical microscope. (B and C) Statistics of tube length and numbers of mashes ( $n=3$ each group). $* P<0.005 ; * * P<0.001 ; * * * p<0.0001$.

expression has been reported involved in many pathological processes, including immune disease, metabolic disease, and cancer. $^{22}$ Recently, numerous reports revealed that miRNAs could regulate the cancer cellular processes, including in endometrial cancer, through targeting oncogenes or suppressive genes. For instance, Chen et al demonstrated that miRNA-505 functions as a tumor suppressor in endometrial cancer by targeting TGF- $\alpha .^{23}$ Gao et $\mathrm{al}^{11}$ reported that miRNA-134 suppresses endometrial cancer stem cells by targeting POGLUT1 and Notch pathway proteins. On the contrary, Wang et $\mathrm{al}^{24}$ reported that delivery of miR-373 decreased LATS2 expression and subsequently activated $\mathrm{Wnt} / \beta$-Catenin pathway activation, which promoted cell proliferation and migration in endometrial cancer cells. Ruan et $\mathrm{al}^{25}$ demonstrated that miR-183 might play an oncogenic role, by regulating MMP-9 expression in endometrial cancer cells. Therefore, identification of endometrial cancer-related miRNAs and their target genes may play a crucial role in the clinical treatment of this high lethality malignancy.

Many recent studies have focused on elucidating the roles of miR-499 in cancer. For instance, Li et al $^{26}$ demonstrated that overexpressed miR-499 inhibits non-small cell lung cancer (NSCLC) growth via suppressing cell proliferation and inducing apoptosis. Liu et $\mathrm{al}^{27}$ reported that microRNA-499 promotes cellular invasion and tumor metastasis in colorectal cancer by targeting FOXO4 and PDCD4. However, there is still a lack of understanding about the mechanisms of miR-499 to promote cancer metastasis in endometrial cancer. In this study, bioinformatics analysis showed that miR-499 was significant decreased in endometrial cancer tissues and cell lines compared with adjacent non-cancer tissues and normal endometrium cell lines. To our knowledge, cancer growth 


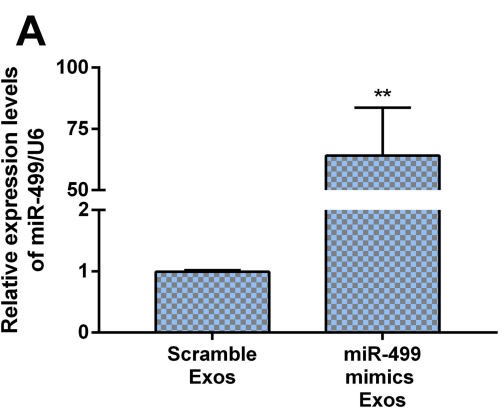

C

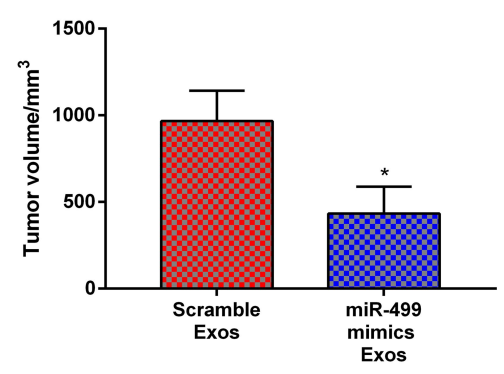

E

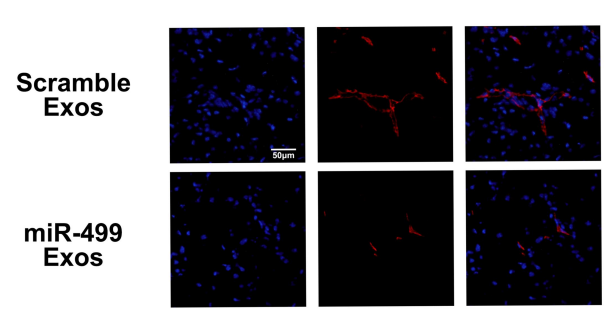

B

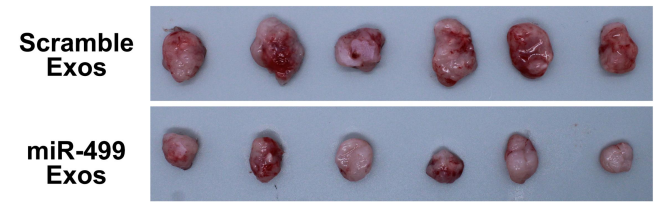

D

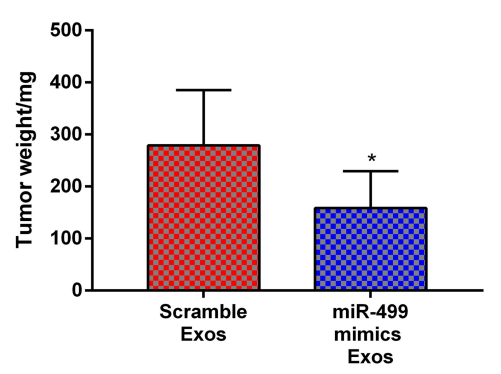

F

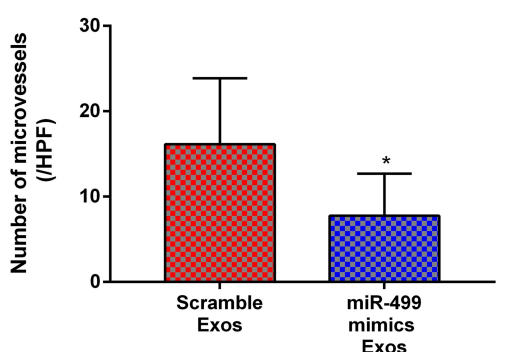

Figure 6 Exosomal miR-499 inhibited tumor growth and angiogenesis in vivo. (A) The expression levels of miR-499 was detected by RT-PCR at day 28 after Ishikawa cell injections ( $n=6$ each group). (B-D) exosomal miR-499 reduced tumor weights and volumes in vivo ( $n=6$ each group). (E) Tumor angiogenesis detected with immunofluorescence staining using an antibody against CD3I ( $\mathrm{n}=6$ each group). (F) Quantitative assessment of capillary density. Data are presented as the means \pm SD. $* P<0.005$; $* * P<0.001$.

A

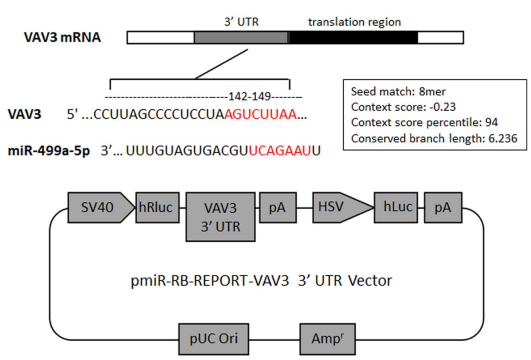

B

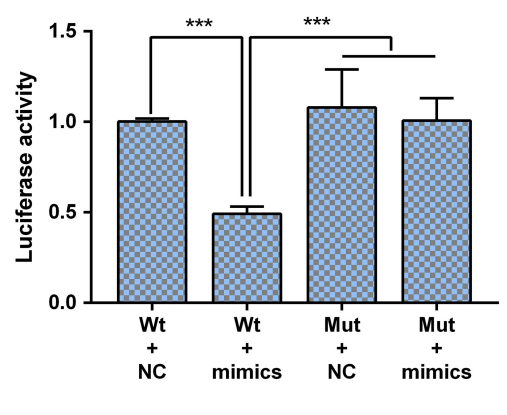

C

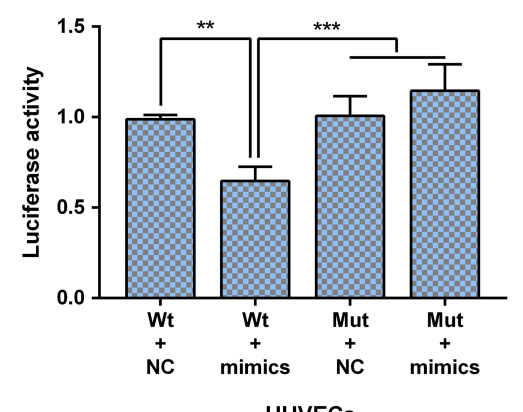

Figure 7 miR-499 inhibits the expression of VAV3 by directly binding its 3'-UTR. (A) Presumptive binding sites of miR-499 on the pmirGLO-VAV3-WT and pmirGLO-VAV3Mut are shown. (B and C) Luciferase activities were detected in Ishikawa and HUVECs ( $\mathrm{n}=3$ each group). Data are presented as the means \pm SD. $* * P<0.00 \mathrm{I}$, $* * * P<0.000 \mathrm{I}$.

and metastasis is usually accompanied by aberrant gene expression. And miR-499 downregulation has been reported in multiple malignancies. These results indicate that miR-499 may play an important role in endometrial cancer growth and metastasis. In addition, our results showed that exosomal miR-499 inhibited endometrial cancer cells proliferation and endothelial cells tube formation in vitro. Tumor cell proliferation and tumor angiogenesis 
are the main events in the process of tumor growth. These findings further clarify the anti-cancer function of miR499 in endometrial cancer. It is well known that miRNA exert a gene regulation effect through binding to the 3 'UTRs of target mRNAs. In the current study, we demonstrated miR-499 target the 3'UTR sequence of VAV3 using DLR. In future investigations, we will focus on rummaging the specific molecular pathway regulated by miR499/VAV3 in endometrial cancer.

Accumulating evidence suggests that MSCs represent potential candidates for regenerative medicine and have possible oncologic applications for the inhibition of tumor progression in some tumor models. Recent evidence has demonstrated that MSC-derived Exos confer similar benefits as MSCs through transporting bioactive factors, particularly miRNAs. ${ }^{28}$ Exos are currently considered as important regulators of cellular communication. Exos have potential applications as drug delivery vehicles and offer several advantages (nonimmunogenic, easy to genetically modify, management, and store) over MSCs. ${ }^{28}$ For Exos-mediated drug delivery, MSCs are better donor cell types, owing to their production efficiency, immune-regulating capacity, and clinical applicability. In this study, we used MSCderived Exos as miR-499 delivery vehicles. We found that Exos loaded with miR-499 can be easily taken up by Ishikawa cells and efficiently up-regulate miR-499 expression in these cells. Our results indicate that employ Exos as transfer vehicles may improve the drug delivery efficiency and may be useful for managing cancers.

In conclusion, we found that miR-499 was downregulated in endometrial cancer tissues and cell lines. Furthermore, exosomal miR-499 inhibits endometrial cancer cell proliferation and endothelial cells tube formation by directly targeting VAV3. Additionally, the evidence presented here suggests that exosomal miR-499 reduced tumor growth and angiogenesis of endometrial cancer in vivo.

\section{Ethics Approval and Consent to Participate}

The experiments were approved by the Ethics Committee of the Affiliated Yantai Yuhuangding Hospital of Qingdao University. Written informed consent was obtained from all patients. The experiments were carried out following the Declaration of Helsinki.

\section{Author Contributions}

All authors made substantial contributions to the conception and design, acquisition of data, or analysis and interpretation of data; took part in drafting the article or revising it critically for important intellectual content; agreed to submit to the current journal; gave final approval of the version to be published; and agree to be accountable for all aspects of the work.

\section{Disclosure}

The authors report no conflicts of interest for this work.

\section{References}

1. Wright JD. What is new in endometrial cancer? Best articles from the past year. Obstet Gynecol. 2016;128(4):904-905. doi:10.1097/ AOG.0000000000001677

2. Hafizz A, Zin RRM, Aziz NHA, Kampan NC, Shafiee MN. Beyond lipid-lowering: role of statins in endometrial cancer. Mol Biol Rep. 2020;47(10):8199-8207. doi:10.1007/s11033-020-05760-5

3. Li Y, Huang C, Kavlashvili T, et al. Loss of progesterone receptor through epigenetic regulation is associated with poor prognosis in solid tumors. Am J Cancer Res. 2020;10(6):1827-1843.

4. Francis SR, Ager BJ, Do OA, et al. Recurrent early stage endometrial cancer: patterns of recurrence and results of salvage therapy. Gynecol Oncol. 2019;154(1):38-44. doi:10.1016/j.ygyno.2019.04.676

5. Bregar AJ, Melamed A, Diver E, et al. Minimally invasive staging surgery in women with early-stage endometrial cancer: analysis of the national cancer data base. Ann Surg Oncol. 2017;24 (6):1677-1687.

6. Connor EV, Rose PG. Management strategies for recurrent endometrial cancer. Expert Rev Anticancer Ther. 2018;18(9):873-885. doi:10.1080/14737140.2018.1491311

7. Polcher M, Rottmann M, Brugger S, et al. Lymph node dissection in endometrial cancer and clinical outcome: a population-based study in 5546 patients. Gynecol Oncol. 2019;154(1):65-71. doi:10.1016/j. ygyno.2019.04.002

8. Iwamoto N, Vettori S, Maurer B, et al. Downregulation of miR-193b in systemic sclerosis regulates the proliferative vasculopathy by urokinase-type plasminogen activator expression. Ann Rheum Dis. 2016;75(1):303-310. doi:10.1136/annrheumdis-2014-205326

9. Rupaimoole R, Slack FJ. MicroRNA therapeutics: towards a new era for the management of cancer and other diseases. Nat Rev Drug Discov. 2017;16(3):203-222.

10. Chen H, Pan H, Qian Y, Zhou W, Liu X. MiR-25-3p promotes the proliferation of triple negative breast cancer by targeting BTG2. Mol Cancer. 2018;17(1):4. doi:10.1186/s12943-017-0754-0

11. Gao Y, Liu T, Huang Y. MicroRNA-134 suppresses endometrial cancer stem cells by targeting POGLUT1 and Notch pathway proteins. FEBS Lett. 2015;589(2):207-214. doi:10.1016/j. febslet.2014.12.002

12. Li BL, Lu W, Qu JJ, Ye L, Du GQ, Wan XP. Loss of exosomal miR-148b from cancer-associated fibroblasts promotes endometrial cancer cell invasion and cancer metastasis. J Cell Physiol. 2019;234 (3):2943-2953. doi:10.1002/jcp.27111

13. Yan H, Sun BM, Zhang YY, et al. Upregulation of miR-183-5p is responsible for the promotion of apoptosis and inhibition of the epithelial-mesenchymal transition, proliferation, invasion and migration of human endometrial cancer cells by downregulating Ezrin. Int J Mol Med. 2018;42(5):2469-2480. 
14. Ushakov DS, Dorozhkova AS, Babayants EV, et al. Expression of microRNA potentially regulated by $\mathrm{AhR}$ and CAR in malignant tumors of the endometrium. Bull Exp Biol Med. 2018;165 (5):688-691. doi:10.1007/s10517-018-4242-3

15. Ando H, Asai T, Koide H, et al. Advanced cancer therapy by integrative antitumor actions via systemic administration of miR-499. J Control Release. 2014;181:32-39. doi:10.1016/j.jconrel.2014.02.019

16. Langrzyk A, Nowak WN, Stepniewski J, et al. Critical view on mesenchymal stromal cells in regenerative medicine. Antioxid Redox Signal. 2018;29(2):169-190. doi:10.1089/ars.2017.7159

17. Phinney DG, Pittenger MF. Concise review: MSC-derived exosomes for cell-free therapy. Stem Cells. 2017;35(4):851-858. doi:10.1002/ stem. 2575

18. Mendt M, Rezvani K, Shpall E. Mesenchymal stem cell-derived exosomes for clinical use. Bone Marrow Transplant. 2019;54(Suppl 2):789-792. doi:10.1038/s41409-019-0616-Z

19. Ma T, Chen Y, Chen Y, et al. MicroRNA-132, delivered by mesenchymal stem cell-derived exosomes, promote angiogenesis in myocardial infarction. Stem Cells Int. 2018;2018:3290372. doi:10.1155/ 2018/3290372

20. Yang K, Li D, Wang M, et al. Exposure to blue light stimulates the proangiogenic capability of exosomes derived from human umbilical cord mesenchymal stem cells. Stem Cell Res Ther. 2019;10(1):358. doi:10.1186/s13287-019-1472-x

21. Brown M, Fan M. Detection of miRNA in cultured cells or xenograft tissues of breast cancer. Methods Mol Biol. 2016;1406:81-88.
22. Khan AQ, Ahmed EI, Elareer NR, Junejo K, Steinhoff M, Uddin S. Role of miRNA-regulated cancer stem cells in the pathogenesis of human malignancies. Cells. 2019;8(8):840. doi:10.3390/cells8080840

23. Chen S, Sun KX, Liu BL, Zong ZH, Zhao Y. MicroRNA-505 functions as a tumor suppressor in endometrial cancer by targeting TGF-alpha. Mol Cancer. 2016;15:11. doi:10.1186/s12943-016-0496-4

24. Wang L, Wang L, Chang W, Li Y, Wang L. MicroRNA-373 promotes the development of esophageal squamous cell carcinoma by targeting LATS2 and OXR1. Int J Biol Markers. 2019;34(2):148-155. doi:10.1177/1724600819827964

25. Ruan H, Liang X, Zhao W, Ma L, Zhao Y. The effects of microRNA-183 promots cell proliferation and invasion by targeting MMP-9 in endometrial cancer. Biomed Pharmacother. 2017;89:812-818. doi:10.1016/j.biopha.2017.02.091

26. Li M, Zhang S, Wu N, Wu L, Wang C, Lin Y. Overexpression of miR-499-5p inhibits non-small cell lung cancer proliferation and metastasis by targeting VAV3. Sci Rep. 2016;6:23100. doi:10.1038/ srep23100

27. Liu X, Zhang Z, Sun L, et al. MicroRNA-499-5p promotes cellular invasion and tumor metastasis in colorectal cancer by targeting FOXO4 and PDCD4. Carcinogenesis. 2011;32(12):1798-1805. doi:10.1093/carcin/bgr213

28. Liu H, Liang Z, Wang F, et al. Exosomes from mesenchymal stromal cells reduce murine colonic inflammation via a macrophage-dependent mechanism. JCI Insight. 2019;4(24). doi:10.1172/jci.insight.131273.

\section{Publish your work in this journal}

Cancer Management and Research is an international, peer-reviewed open access journal focusing on cancer research and the optimal use of preventative and integrated treatment interventions to achieve improved outcomes, enhanced survival and quality of life for the cancer patient.
The manuscript management system is completely online and includes a very quick and fair peer-review system, which is all easy to use. Visit http://www.dovepress.com/testimonials.php to read real quotes from published authors. 